\title{
The Wnt inhibitor LGK-974 enhances radiosensitivity of HepG 2 cells by modulating Nrf2 signaling
}

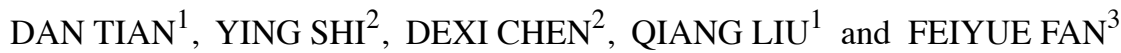 \\ ${ }^{1}$ Institute of Radiation Medicine, Chinese Academy of Medical Sciences (CAMS) and Peking Union Medical College \\ (PUMC), Tianjin 300192; ${ }^{2}$ Beijing Institute of Hepatology, Beijing Youan Hospital, Capital Medical University, \\ Beijing 100069; ${ }^{3}$ Institute of Laboratory Animal Sciences, Peking Union Medical College (PUMC) \\ and Chinese Academy of Medical Sciences (CAMS), Beijing 100069, P.R. China
}

Received October 22, 2016; Accepted May 15, 2017

DOI: $10.3892 /$ ijo.2017.4042

\begin{abstract}
Nuclear factor (erythroid-derived 2)-like 2 (NRF2) is a master regulator of antioxidant and detoxification activities that can eliminate reactive oxygen species (ROS) produced via irradiation. However, Nrf2 overexpression in liver cancer cells may cause both radioresistance and chemoresistance. Reducing Nrf2 levels can enhance the radiosensitivity of HepG2 cells. Wingless/int-3A (Wnt3A) is a Wnt family protein that mainly activates the canonical Wnt signaling pathway. Recent studies showed that the Axin1-GSK-3 $\beta$ protein complex, a component of the canonical Wnt signaling pathway, can capture Nrf2 and facilitate its ubiquitination and proteasomal degradation in the cytoplasm. This protein complex is degraded upon activation of the Wnt signaling pathway. In the present study, we treated HepG2 cells with the Wnt3A inhibitor LGK-974, an effective and specific PORCN inhibitor that can prevent the formation of a proper folding of the Wnt protein in the endoplasmic reticulum. We found that HepG2 cells became more sensitive to radiation with increasing LGK-974 concentrations. Upon 2 Gy or 4 Gy irradiation, the cells treated with LGK-974 more frequently underwent apoptosis and grew less rapidly. PCR and western blot results showed that inhibiting the secretion of Wnt3A blocked the Wnt signaling pathway and prevented Nrf2 signaling. Notably, the Wnt inhibitor may serve as a radiosensitizing drug.
\end{abstract}

Correspondence to: Dr Qiang Liu, Institute of Radiation Medicine, Chinese Academy of Medical Sciences (CAMS) and Peking Union Medical College (PUMC), Tianjin 300192, P.R. China

E-mail: liuqiang@irm-cams.ac.cn

Dr Feiyue Fan, Institute of Laboratory Animal Sciences, Chinese Academy of Medical Sciences (CAMS) and Peking Union Medical College (PUMC), Beijing 100069, P.R. China

E-mail: faithyfan@hotmail.com

Key words: LGK-974, HepG2 cells, wingless/int-3A, nuclear factor (erythroid-derived 2)-like 2, reactive oxygen species, radiation, radiosensitivity

\section{Introduction}

Liver cancer is a malignant cancer (1), and radiotherapy is a common means of prolonging the life of patients with middle- to late-stage cancer (2). Radiogenic reactive oxygen species (ROS) induce cancer cell death (3). The transcription factor nuclear factor (erythroid-derived 2)-like 2 (Nrf2) is a master regulator of oxidative and xenobiotic metabolism (4) that is sensitive to ROS (5). Nrf2 controls the transcription and expression of $\sim 1 \%$ of all human genes; Nrf2-regulated genes participate in biotransformation reactions, redox status, energy metabolism and proteostasis (6-9). The expression products of Nrf2-targeted genes, such as antioxidant enzymes, can relieve the oxidative stress generated by ionizing radiation in cancer cells (10-12), leading to resistance to cancer radiotherapy. Under normal conditions, Nrf2 interacts with Kelch-like ECH-associated protein 1 (Keap1) in the cytoplasm (13). KEAP1 interacts with two regions of NRF2 (amino-acid sequences DLG and ETGE) located in the N-terminal Neh2 domain to direct ubiquitination of NRF2 by the Cullin-3/Rbx1 complex and the proteasomal degradation of NRF2 $(14,15)$ (Fig. 1). Upon oxidation of KEAP1, NRF2 escapes degradation, enters the nucleus, and then binds to the Maf protein, which targets antioxidant response element (ARE) genes to initiate downstream antioxidant gene transcription (16-19). For cancer radiotherapy, we aimed to inhibit the Nrf2-ARE signaling pathway. We found that the Wnt inhibitor LGK-974 can hinder the entrance of Nrf2 into the nucleus of HepG2 cells. Another report indicated that NRF2 participates in the formation of an Axin1/GSK-3/b-TrCP protein complex that is regulated by WNT-3A in liver cells (20).

LGK-974 inhibits Wnt signaling pathways by blocking secretion of the Wnt3A protein in HepG2 cells. The canonical Wnt signaling pathway is initiated upon binding of secreted Wnt3A to the receptors LRP5/6 and Frizzled, which are embedded in the cell membrane $(21,22)$. LRP5/6 has five contiguous PPPSPXS motifs, and Axin can bind to a phosphorylated PPPSPXS motif, GSK-3 $\beta$ and CK1 simultaneously $(23,24)$. The association between Axin and LRP5/6 ultimately contributes to the degradation of AxinGSK3 $\beta$-CK1-APC protein complexes. Thus, $\beta$-catenin avoids degradation by Axin-GSK3 $\beta-\mathrm{CK} 1-\mathrm{APC}$ complexes. In the 
cytoplasm, $\beta$-catenin accumulates and enters the nucleus to activate different genes that regulate cellular proliferation and differentiation (25).

LGK-974inhibits porcupine(PORCN), anO-acyltransferase in the endoplasmic reticulum required for the secretion of Wnt, and therefore inhibits endogenous Wnt signaling (26). In the presence of LGK-974, Wnt3A palmitoylation does not occur, and the Wnt protein cannot form its proper conformation (27-29). Ultimately, Wnt3A becomes stranded in the cytoplasm. Given this promising finding, we suspect that LGK-974 might enhance the efficacy of radiotherapy by inhibiting $\mathrm{Nrf} 2$.

\section{Materials and methods}

Chemicals and reagents. High-glucose Dulbecco's modified Eagle's medium (DMEM) and penicillin/streptomycin were purchased from HyClone Laboratories, Inc., (Logan, UT, USA). Fetal bovine serum (FBS) was obtained from Gibco-BRL (Grand Island, NY, USA). LGK-974 was obtained from Selleck Chemicals (Houston, TX, USA). Trypsin, DEPCtreated water, dimethyl sulfoxide (DMSO), RIPA lysis buffer, Giemsa stain and other reagents were purchased from Beijing Solarbio Life Sciences (Beijing, China). MTT and $\mathrm{H}_{2}$ DCF-DA were obtained from Sigma-Aldrich (St. Louis, MO, USA). Primary antibodies including rabbit/mouse antibodies against Wnt3A, Nrf2, $\beta$-catenin, APC-6, NQO-1, HO-1 and survivin were purchased from Abcam (Cambridge, MA, USA), and the anti-Keap-1 antibody was purchased from Santa Cruz Biotechnology (Santa Cruz, CA, USA). Horseradish peroxidase-conjugated anti-rabbit IgG and anti-mouse IgG were obtained from ZSGB-Bio (Beijing, China).

Cell culture. Cells were cultured in high-glucose DMEM containing $10 \% \mathrm{FBS}$ and $100 \mathrm{U} / \mathrm{ml}$ penicillin/streptomycin at $37^{\circ} \mathrm{C}$ in a humidified $\mathrm{CO}_{2}$ incubator.

LGK-974 treatment and irradiation. The cells were divided into four groups: group a, control; group b, LGK-974 alone; group c, irradiation alone; and group d, LGK-974 + irradiation. The LGK-974 and LGK-974 + irradiation groups were treated with $0.1,1,5$ or $10 \mu \mathrm{M}$ LGK-974. After $24 \mathrm{~h}$, the irradiation and LGK-974 + irradiation groups were exposed to $2 \mathrm{~Gy}$ or 4 Gy doses of $137 \mathrm{Cs} \gamma$-rays at a dose rate of 1.02 Gy per minute in an exposure instrument (Atomic Energy of Canada Ltd., Chalk River, ON, Canada). LGK-974 was dissolved in the solvent DMSO according to the manufacturer's instructions. We dissolved $5 \mathrm{mg}$ of LGK-974 into $1.2612 \mathrm{ml}$ of DMSO, obtaining a $10 \mathrm{mM}$ LGK-974 starting solution. The experimental concentrations, such as $0.1,1,5$ and $10 \mu \mathrm{M}$, were generated by diluting the $10 \mathrm{mM}$ LGK-974 starting solution with culture medium.

HepG2 cell counts, growth curve and clone formation assay. Cells were seeded in 12-well plates at a density of $3 \times 10^{4}$ cells/well in triplicate and then treated with the indicated concentrations of LGK-974 and the indicated doses of irradiation, followed by 6 days in culture. The medium was replaced with the identical fresh media every $24 \mathrm{~h}$, and the number of surviving cells was counted daily using trypan blue and a hemocytometer. HepG2 cells were plated at the same densities (100 cells/well) in 12-well plates in triplicate, treated with $1.0 \mu \mathrm{M}$ LGK-974 for $24 \mathrm{~h}$, and then exposed to the indicated doses of $137 \mathrm{Cs}$ radiation $(1.02 \mathrm{~Gy} / \mathrm{min})$. Two hours after irradiation, the medium was replaced with fresh medium without any drug. The cells were then incubated for $\sim 8$ days, with changes of medium every 2 days. Finally, the cells were subjected to Giemsa staining and colonies containing $>50$ cells were counted.

Cell viability assay. HepG2 cell viability was determined using an MTT assay, which involves the reduction of yellow MTT by mitochondrial succinate dehydrogenase in viable cells. HepG2 cells were cultured in a 96-well plate $(5,000$ cells/well) for $24 \mathrm{~h}$. Then, the experimental groups were starved in serumfree media overnight. A series of concentrations of LGK-974 $(0.1,1$ and $10 \mu \mathrm{M})$ were added to each well for groups $\mathrm{b}$ and $\mathrm{d}$, which were then cultured for an additional $24 \mathrm{~h}$. Group c and group $\mathrm{d}$ were exposed to $2 \mathrm{~Gy}$ or $4 \mathrm{~Gy} \gamma$-irradiation and then incubated with $5 \mathrm{mg} / \mathrm{ml}$ MTT for $4 \mathrm{~h}$ in the dark. The supernatant was removed, and the formazan crystals in each well were dissolved in $150 \mu \mathrm{l}$ of DMSO with shaking for $15 \mathrm{~min}$ at room temperature in the dark. The absorbance at $562 \mathrm{~nm}$ was measured using a microplate reader. Cell viability was expressed as a percentage relative to the vehicle-treated control cells.

Flow cytometry for apoptosis assays and detection of intracellular ROS. HepG2 cells in the logarithmic growth phase were treated with $0.1,1$ or $10 \mu \mathrm{M}$ LGK-974 and exposed to $2 \mathrm{~Gy}$ or $4 \mathrm{~Gy} \gamma$-irradiation. Cells in both the control group and the experimental groups were collected $12 \mathrm{~h}$ after the treatment. HepG2 cells were prepared and evaluated according to the instructions with the Annexin V-FITC/PI apoptosis detection kit. Cell apoptosis assays were performed using flow cytometry (BD Biosciences, San Jose, CA, USA). Intracellular ROS levels were measured using $\mathrm{H}_{2}$ DCFH-DA (Molecular Probes/ Invitrogen, Waltham, MA, USA). HepG2 cells were treated with or without $10 \mu \mathrm{M}$ LGK-974 and $10 \mu \mathrm{l} \mathrm{H}_{2} \mathrm{O}_{2} 6 \mathrm{~h}$ prior to irradiation and then treated with $10 \mu \mathrm{M} \mathrm{H}_{2} \mathrm{DCFH}-\mathrm{DA}$ for $30 \mathrm{~min}$. Next, the cells were washed with phosphate-buffered saline (PBS), followed by trypsinization. After detachment, the cells were collected, washed twice and resuspended in $500 \mu 1$ of PBS. We used a flow cytometer at excitation/emission wavelengths of $488 / 525 \mathrm{~nm}$ to measure the fluorescence.

Immunofluorescence. HepG2 cells $\left(2.0 \times 10^{5}\right.$ cells/well) were cultured in 24-well plates on sterilized coverslips, treated with or without $10 \mu \mathrm{M}$ LGK-974/ and exposed to $4 \mathrm{~Gy}$. After $6 \mathrm{~h}$, these cells were washed with PBS and fixed with $4 \%$ paraformaldehyde for $20 \mathrm{~min}$ at room temperature. Afterwards, the cells were washed 3 times with PBS for $5 \mathrm{~min}$ and permeabilized in $0.3 \%$ Triton X-100/PBS for $15 \mathrm{~min}$. Next, the cells were subjected to immunodetection. All washes were performed 3 times with PBS for $5 \mathrm{~min}$. The cells were incubated with $10 \%$ goat serum/PBS for $30 \mathrm{~min}$ at room temperature and then incubated with the polyclonal anti-Nrf2 antibody at 1:1,000 in $10 \%$ goat serum/PBS overnight at $4^{\circ} \mathrm{C}$. Then, the cells were washed and incubated with the FITC-conjugated secondary anti-rabbit $\mathrm{IgG}$ antibody diluted 1:100 in 10\% goat serum/ PBS for $1 \mathrm{~h}$. Finally, the cells were washed and incubated with 
$50 \mu \mathrm{l}$ of $0.5 \mu \mathrm{g} / \mathrm{ml}$ DAPI for $5 \mathrm{~min}$ at room temperature to stain the nuclei. The fluorescence images were captured using a fluorescence microscope.

Quantitative reverse-transcription PCR. HepG2 cells were harvested, and total RNA was isolated using TRIzol ${ }^{\circledR}$ reagent (Invitrogen/Life Technologies). cDNA was reverse-transcribed with $5 \mu \mathrm{g}$ of total RNA using oligo(dT) primers and M-MLV reverse transcriptase (Promega, Fitchburg, WI, USA). The primer sequences were as follows: 5'-CCACCAGCAGCGAC TCTGA-3' and 5'-GCAGAAGGTGATCCAGACTC-3' as forward and reverse primers for C-myc, respectively; 5'-GTGG CAGTGGCTCCATGTACTC-3' and 5'-CTTGGAAGCCAC AGAAATGCAG-3' as forward and reverse primers for NQO-1, respectively; 5'-TTGCCAGTGCCACCAAGTTC-3' and 5'-TCAGCAGCTCCTGCAACTCC-3' as forward and reverse primers for $\mathrm{HO}-1$, respectively; and 5'-CTGCACCACC AACTGCTTAG-3' and 5'-AGGTCCACCACTGACACGTT-3 as forward and reverse primers for GAPDH, respectively.

Western blot analysis. Cancer cells were washed twice with ice-cold PBS (pH 7.4), harvested in cell lysis buffer at $4^{\circ} \mathrm{C}$ for 20-30 min and centrifuged at $12,000 \mathrm{rpm}$ at $4^{\circ} \mathrm{C}$ for $15 \mathrm{~min}$ to obtain total protein lysates for immunoblot analysis. The protein concentrations were quantified using the BCA protein assay reagent according to the manufacturer's protocols. Equal amounts of protein were loaded onto $12 \%$ SDS-polyacrylamide gels for electrophoresis; then, the proteins were transferred onto a PVDF membrane (Bio-Rad Laboratories, Hercules, CA, USA) for $1 \mathrm{~h}$ using a semi-dry transfer system (Bio-Rad Laboratories). The membrane was blocked with $5 \%$ non-fat milk in PBST buffer (0.1\% Tween-20 in PBS) for $2 \mathrm{~h}$ at room temperature and then incubated with the appropriate primary antibodies overnight. After hybridization with a primary antibody, the membrane was washed three times with TBST and then incubated with anti-mouse or anti-rabbit IgG horseradish peroxidase-conjugated secondary antibodies for $50 \mathrm{~min}$ at room temperature, followed by three washes with TBST. Finally, the immunoreactive bands were visualized using enhanced chemiluminescence reagents.

Single-cell gel assay (comet assay). After exposure to $4 \mathrm{~Gy}$ irradiation, cells were digested and collected immediately, washed twice with PBS, and suspended in PBS at a concentration of $4 \times 10^{5}$ cells $/ \mathrm{ml}$. The comet slides were coated with $0.75 \%$ normal-melting-point agarose/PBS. Once the first layer of normal-melting-point agarose was coagulated, a mixture of $70 \mu \mathrm{l}$ low-melting-point agarose/PBS and $30 \mu 1$ of cell suspension was applied as the second layer. The comet slides were then immersed in cold fresh lysis solution $(2.5 \mathrm{M} \mathrm{NaCl}, 10 \mathrm{mM}$ Tris base, $1 \% \mathrm{~N}$-sodium lauryl sarcosinate, $30 \mathrm{mM} \mathrm{Na}{ }_{2}$ EDTA, $10 \%$ DMSO, $1 \%$ Triton X-100) for $2.5 \mathrm{~h}$ at $4^{\circ} \mathrm{C}$. Next, the comet slides were immersed in TBE buffer for $20 \mathrm{~min}$ in a horizontal electrophoresis tank and electrophoresis was performed at $30 \mathrm{~V}$ for $20 \mathrm{~min}$ in TBE buffer, followed by neutralization for $20 \mathrm{~min}$. The slides were then rinsed twice with PBS and stained with ethidium bromide. Finally, the comet slides were viewed under a fluorescence microscope, and data were collected using a digital imaging system and analyzed with CASP software (CaspLab, Wroclaw, Poland).
Statistical analysis. Each experiment was performed at least 3 times, and the data are expressed as the means \pm standard deviation (SD). For comparisons between three or more groups, one-way analysis of variance was used, followed by Tukey's multiple comparison tests. Statistical analysis was performed using the GraphPad Prism software, version 5.0 (GraphPad Software, Inc., San Diego, CA, USA).

\section{Results}

Comparison of the radiosensitivity of three human hepatic carcinoma cell lines. To evaluate the Nrf2 protein levels between three liver cell lines, we performed western blot assays on nuclear and cytoplasmic proteins. To test the effects of radiation on each cell line, we performed 3-(4,5-dimethylthiazol-2-yl)-2,5-diphenyltetrazolium bromide (MTT) assays. The Nrf2 levels in total protein samples were not notably different between the three hepatoma cell types under normal culture conditions, although SMMC-7721 cells had the highest Nrf2 protein levels (Fig. 1A and C). However, in the nucleus, the Nrf2 level in HepG2 cells was the lowest of the three cell lines, followed by Hep3B cells and then SMMC-7721 cells (Fig. 1B and D). This result showed that Nrf2 is primarily localized to the cytoplasm in HepG2 cells, is equally distributed between the cytoplasm and nucleus in Hep3B cells, and is predominantly localized to the nucleus in SMMC-7721 cells. To test the effects of irradiation on these three cell lines, we exposed HepG2, Hep3B and SMMC-7721 cells to 2 Gy irradiation. Then, western blot assays were performed to examine the Nrf2 content. The results showed that Nrf2 expression was increased in both total protein samples and nuclear fractions (Fig. 1E and F); in addition, the level of Nrf2 in the nucleus of HepG2 cells was added by the greatest extent among the three cell lines (Fig. 1F and $\mathrm{H}$ ). Based on the results following exposure of cells to $0,2,4$ or 6 Gy irradiation, HepG2 and Hep3B cells were clearly more sensitive to irradiation than SMMC-7721 cells (Fig. 1I). The above results show that SMMC-7721 is not sensitive to radiation and that the antioxidative transcription factor Nrf2 is primarily localized to the nucleus in SMMC-7721 cells. The radioresistant effect of Nrf2 is primarily attributable to its function in the nucleus. It is very likely that nuclear Nrf2 plays a role in the protection against radiation once this protein sufficiently accumulates in the nucleus. Irradiation had the greatest influence on Nrf2 distribution in HepG2 cells and it can push Nrf2 into HepG2 cell nucleus. To establish whether the Wnt3A inhibitor LGK-974 can prevent the entry of Nrf2 into the nucleus and to clearly distinguish cytoplasmic Nrf2 from nuclear Nrf2, we selected HepG2 cells as the model for our further research.

LGK-974 increases the radiosensitivity of HepG2 cells. To suppress interference caused by the solvent DMSO, we performed growth curve assays and MTT assays on HepG2 cells treated with LGK-974 solution or its solvent DMSO. We found that DMSO had little impact on the cells. The results were comparable between the untreated control and DMSO groups, and both showed differences compared with the LGK-974 group (Fig. 2A-C). To clarify the radiosensitizing effect of LGK-974 on HepG2 cells, we obtained cell growth curves by performing MTT assays and apoptosis assays via 
A

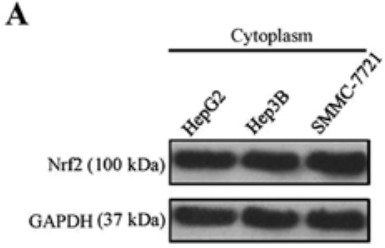

B

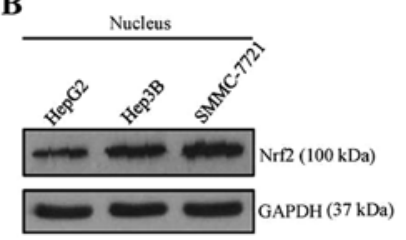

C

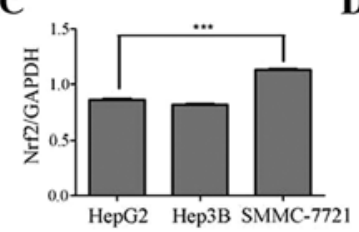

D

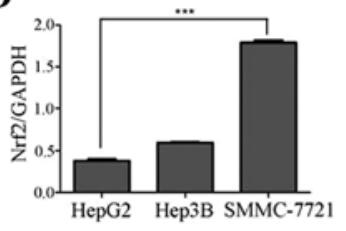

F

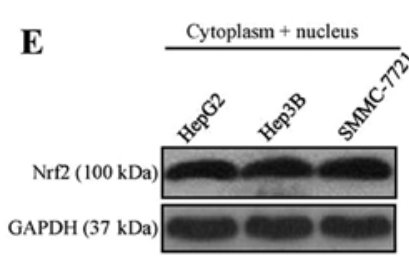

G

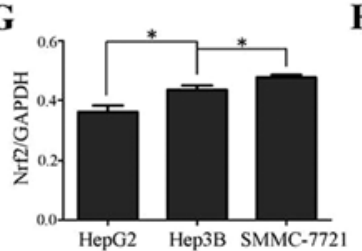

$\mathbf{H}$

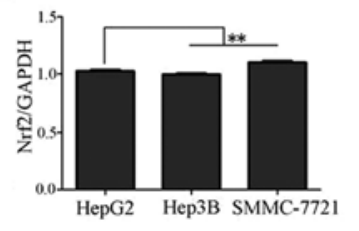

I

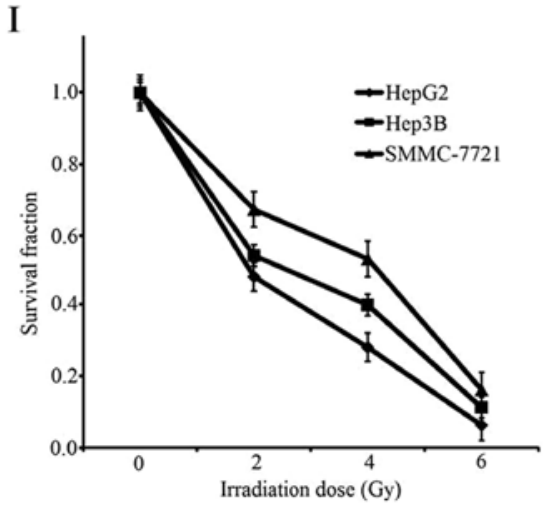

Figure 1. Nrf2 protein levels in the cytoplasm and nucleus of three types of hepatoma cells and the differences in radiosensitivity between cell types. (A and C) Total protein extracts of HepG2, Hep3B and SMMC-7721 cells cultured under normal conditions were evaluated using western blot assays, and Nrf2 protein levels were compared between cell types. (B and D) Nrf2 protein levels in nuclei of HepG2, Hep3B and SMMC-7721 cells; ${ }^{* * * *} \mathrm{P}<0.001$ (E and G) HepG2, Hep3B and SMMC-7721 cells were exposed to 2 Gy irradiation, followed by total protein extraction for analysis of the Nrf2 protein levels. $(\mathrm{F}$ and $\mathrm{H}) \mathrm{Nrf2}$ protein levels in the nuclear fraction of HepG2, Hep3B and SMMC-7721 cells after exposed to $2 \mathrm{~Gy}$. (I) Proportion of surviving cells as determined via an MTT assay. ${ }^{* *} \mathrm{P}<0.01,{ }^{*} \mathrm{P}<0.05$.

flow cytometry. To observe radiosensitivity of cells treated with LGK-974 visually, we performed a clone formation assay. The results of these experiments showed that LGK-974 increased the sensitivity of HepG2 cells to irradiation. HepG2 cells were treated with LGK-974 alone, ionizing radiation alone, or both the drug and the radiation. We counted the number of cells before the treatment. After $2 \mathrm{~Gy}, 4 \mathrm{~Gy}, 6 \mathrm{~Gy}$ or 8 Gy irradiation, the number of viable cells decreased substantially, and the proliferation rate decreased as the irradiation dose increased (Fig. 2D). LGK-974 suppresses HepG2 cell growth and exerts cytotoxic effects (Fig. 2E). The cells grew until five days after LGK-974 treatment; thereafter, the cell growth rate decreased. We found that LGK-974 increased the radiosensitivity of HepG2 cells. The cells treated with $10 \mu \mathrm{M}$ LGK-974 and exposed to 4 Gy irradiation grew the most slowly of all tested cells (Fig. 2F). The results of the clone formation assay showed that LGK-974 enhanced the radiosensitivity of HepG2 cells (Fig. 2I and J). In apoptosis assays conducted via flow cytometry, the HepG2 cells in the experimental groups were cultured in 1 or $10 \mu \mathrm{M}$ LGK-974. After $24 \mathrm{~h}$, the exposure groups received 4 Gy irradiation; $4 \mathrm{~h}$ later, the cells were collected for apoptosis assays. The HepG2 cells in the combined irradiation and LGK-974 group exhibited the highest apoptosis rate (Fig. 2G and H). In MTT assays, after culturing the cells in different concentrations of LGK-974 for $24 \mathrm{~h}$, we determined the proportion of surviving cells. The highest rates of cell death occurred in HepG2 cells treated with 10 or $50 \mu \mathrm{M}$ LGK-974. However, the proportion of surviving cells was comparable between these two groups. The survival rate of the $50 \mu \mathrm{M}$ LGK-974 group was very low, and the number of cells was too low to perform any additional experiments. Given the above results, we chose $10 \mu \mathrm{M}$ LGK-974 for subsequent experiments.

LGK-974 inhibits the activation of Nrf2 in HepG2 cells after exposure to irradiation. To demonstrate the effects of LGK-974, we performed western blot assays and RT-PCR assays. HepG2 cells were cultured in different concentrations of LGK-974. After $24 \mathrm{~h}$, we extracted total protein samples for western blot assays (Fig. 3A). The expression of Nrf2 and its downstream genes HO-1 and NQO-1 clearly decreased as the LGK-974 concentration increased (Fig. 3A, B, D and E). However, the levels of Wnt3A and survivin increased with increasing LGK-974 concentrations (Fig. 3A and C). This phenomenon explained how LGK-974 could restrict Wnt3A to the cytoplasm, thus, preventing the secretion of large amounts of Wnt3A from HepG2 cells. After the cells were exposed to $4 \mathrm{~Gy}$ irradiation, the Wnt3A level declined. LGK-974 treatment caused a gradual increase in the Wnt3A content (Fig. 3G and J). This finding confirmed the above hypotheses. The decrease in survivin expression demonstrated the cytotoxicity of LGK-974 (Fig. 3A and F). To evaluate the effects of LGK-974 and irradiation on HepG2 cells, we treated the cells with different concentrations of LGK-974 for $24 \mathrm{~h}$. Then, the cells were exposed to $4 \mathrm{~Gy}$ radiation. After $8 \mathrm{~h}$, we extracted total protein samples for western blot assays (Fig. 3G). After 4 Gy irradiation, the Nrf2 level increased, indicating that irradiation can increase oxidative stress in cells. To handle this stress, oxidative activity and protein synthesis of the transcription factor Nrf2 protein increased. However, the Nrf2 concentration decreased in the presence of LGK-974 (Fig. 3G and H). In cells treated with $1 \mu \mathrm{M}$ LGK-974, the Nrf2 concentration was $<0.1 \mu \mathrm{M}$; however, this concentration remained lower than the Nrf 2 concentrations in the control cells and the radiation-exposed cells. The content of Nrf2 protein in HepG2 cells clearly decreased. However, at low concentrations of LGK-974, this trend was not apparent, or there was 
A

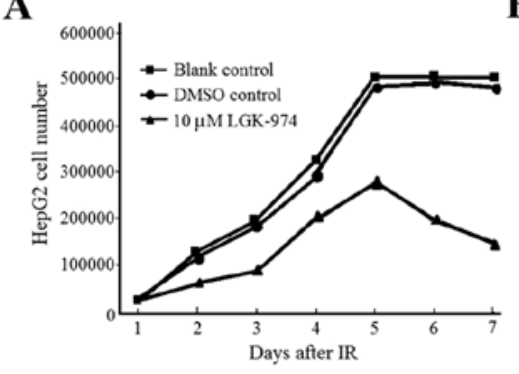

B

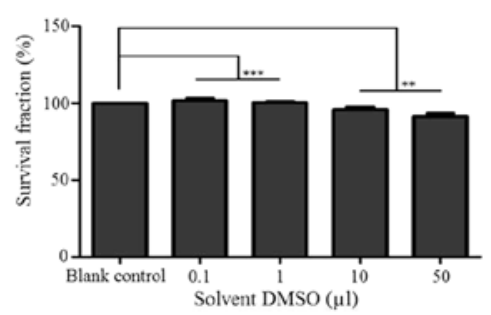

C

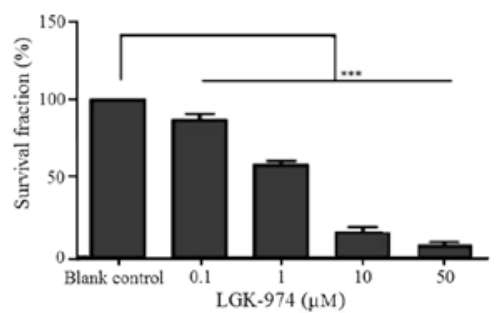

D

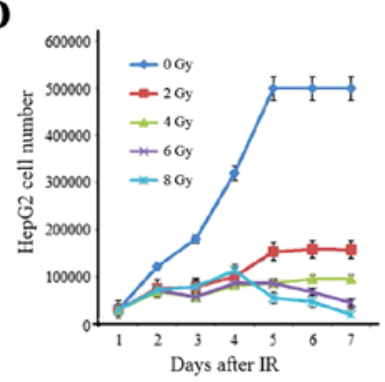

G

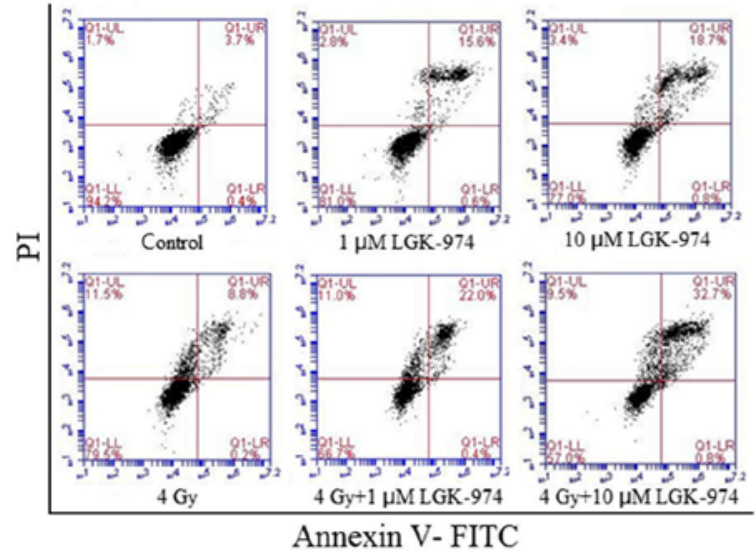

F

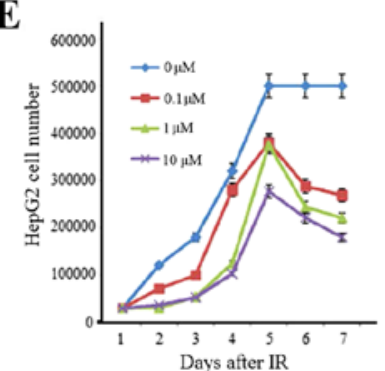

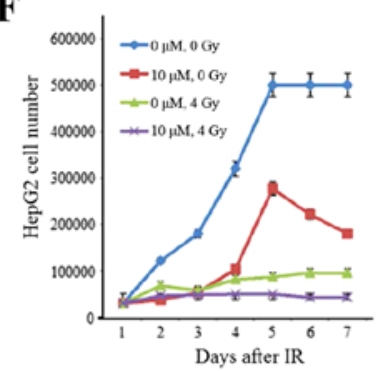

$\mathbf{J}$

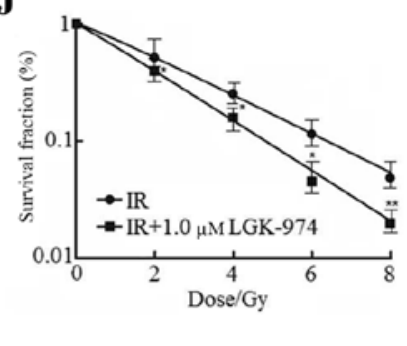

H
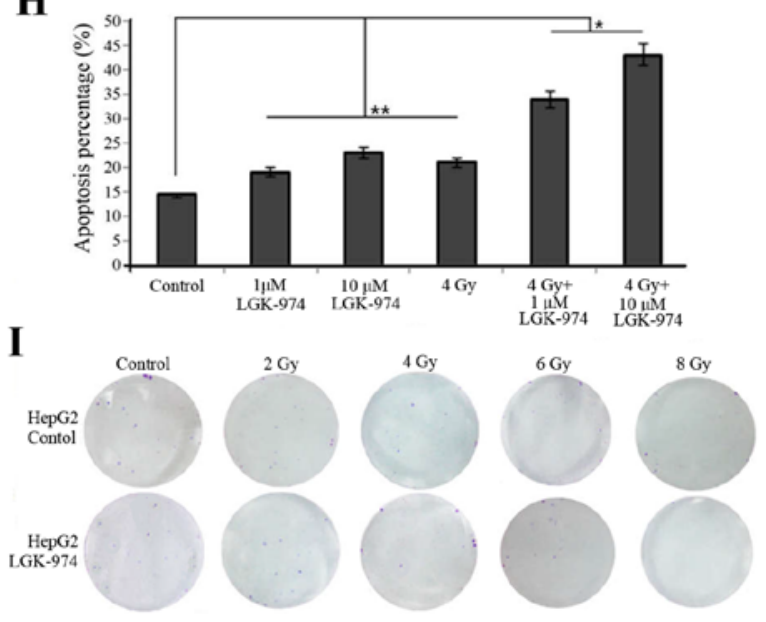

Figure 2. The Wnt3A inhibitor LGK-974 increases the radiosensitivity of HepG2 cells. (A) Growth rate of HepG2 cells treated with LGK-974 solution or its solvent DMSO. The number of HepG2 cells was counted at 7 days. The results were identical between the solvent DMSO group and the blank control group, and HepG2 cell proliferation and survival rates decreased as the LGK-974 concentration increased. (B and C) HepG2 cells were incubated with different concentrations of LGK-974 or a corresponding amount of solvent DMSO for $48 \mathrm{~h}$; then, MTT assays were performed. Survival rate of HepG2 cells under different concentrations. The cell survival rate changed so little as the DMSO concentration increased. The cell survival rate decreased as the LGK-974 concentration increased. In the presence of $50 \mu \mathrm{M}$ LGK-974, nearly all cells died. (D and E) Growth rate of HepG2 cells after exposure to different doses of irradiation and different concentrations of LGK-974. Radiation had a strong influence on cell growth. Four days after 6 Gy or 8 Gy irradiation, all HepG 2 cells gradually died. Most cells survived after 2 Gy or 4 Gy irradiation. (E) HepG2 cell proliferation and survival capability decreased as the LGK-974 concentration increased. The cells gradually died after the fifth day. The HepG2 cells received $10 \mu \mathrm{M}$ LGK-974 had the lowest growth rate and the maximum mortality. (F) Growth rate of HepG 2 cells after exposure to $4 \mathrm{~Gy}$ or $10 \mu \mathrm{M}$ LGK-974. The cells without any processing had the highest growth rate. The cells that received both $10 \mu \mathrm{M}$ LGK-974 and 4 Gy had the lowest growth rate. (G and H) Cell apoptosis after different treatments. The cells in the LGK-974 and 4 Gy radiation treatment group exhibited the highest apoptosis rate. (I and J) HepG2 cells were cultured in 12-well culture plates at 100 cells/well, treated with LGK-974 solution for $24 \mathrm{~h}$, and then exposed to $2,4,6$, or 8 Gy irradiation. At 8 days, the results were analyzed. ${ }^{* * *} \mathrm{P}<0.001,{ }^{* *} \mathrm{P}<0.01,{ }^{*} \mathrm{P}<0.05$.

interference caused by other factors in these cells. The NQO-1 level was strongly affected by irradiation (Fig. 3A and I). The level of $\beta$-catenin after ionizing radiation and treatment with 0.1 or $1 \mu \mathrm{M}$ LGK-974 tended to be increased but was decreased by treatment with 5 or $10 \mu \mathrm{M}$ LGK-974 (Fig. 3A and $\mathrm{K})$. This result verified that ionizing radiation promotes the secretion of Wnt3A and that Wnt3A stimulated the canonical Wnt pathway. As the LGK-974 concentration increased, the canonical Wnt pathway was further inhibited. The level of $\beta$-catenin decreased gradually with increasing LGK-974 concentrations. To reveal the effect of the solvent DMSO on the experimental results, we performed western blot assays on cells treated with DMSO alone. The results demonstrated that DMSO had nearly no effect on Wnt3A and Nrf2 expression, and the results between the blank control and DMSO control groups were identical (Fig. 3L-N). Using DMSO as a control, we also obtained similar results that LGK-974 decreased Nrf2 levels and inhibited NQO-1 and HO-1 expression (Fig. 3O-R). The mRNA levels of HO-1 and NQO-1 increased substantially after 4 Gy irradiation and then decreased due to LGK-974 
A

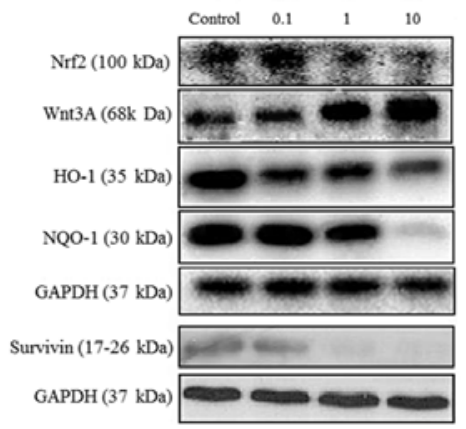

B

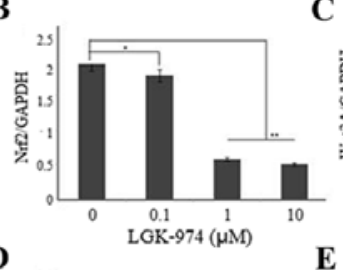

D

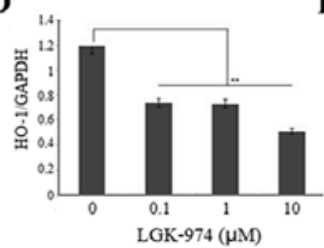

C

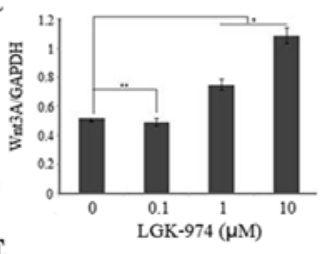

$\mathbf{H}$
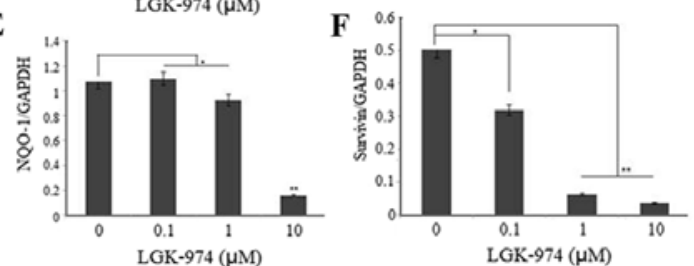

I

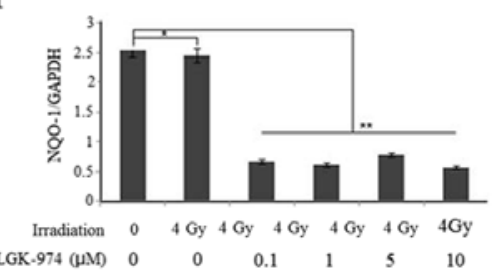

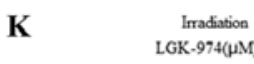
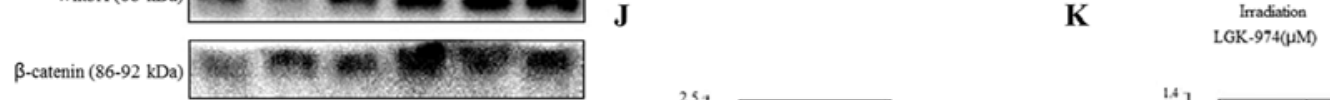

$\mathbf{J}$
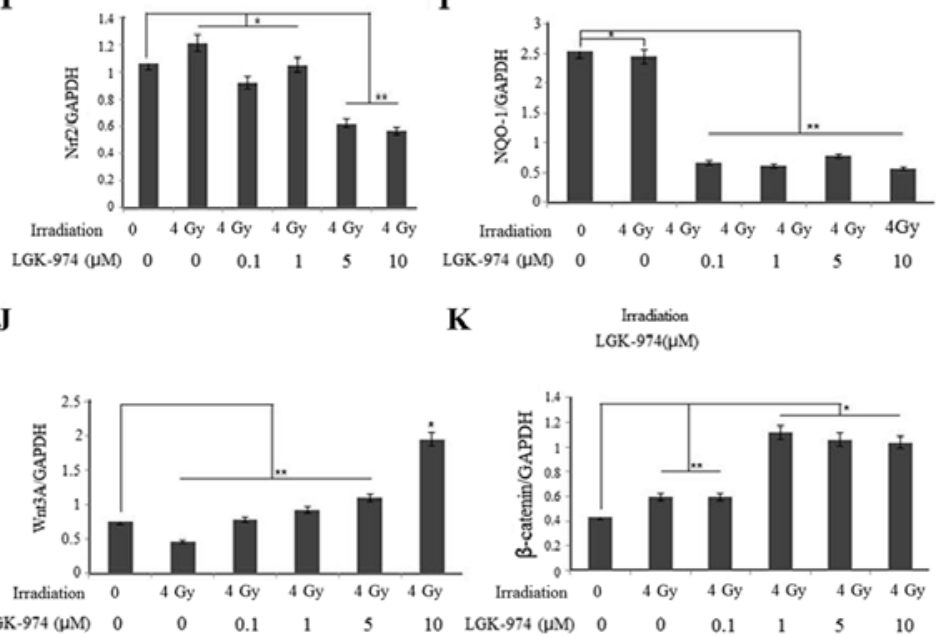

Figure 3. The Wnt3A inhibitor LGK-974 can modulate the Nrf2 signaling pathway. (A) HepG2 cells were treated with LGK-974 at the indicated concentrations and collected after $24 \mathrm{~h}$. Then, we extracted total protein samples for western blot analysis. (B-F) Relative levels of Nrf2, Wnt 3A, HO-1, NQO-1 and survivin in HepG2 cells after treatment with different concentrations of LGK-974. (G) HepG2 cells were treated with different concentrations of LGK-974. After 20 h, the cells were exposed to $4 \mathrm{~Gy}$ radiation. After $8 \mathrm{~h}$, we collected the cells for western blot analysis. (H-K) Nrf2, NQO-1, Wnt3A and $\beta$-catenin levels in HepG2 cells under the different treatments described above.

treatment (Fig. 3S). This result demonstrated that irradiation can activate the Nrf2 pathway and that LGK-974 can inhibit the Nrf2 pathway. The trends in the C-myc and survivin mRNA levels were identical to those for the HO-1 and NQO-1 proteins (Fig. 3T). Irradiation induces the translocation of $\mathrm{Nrf} 2$ into the cell nucleus, but LGK-974 weakens this phenomenon and inhibits Nrf2 expression (Fig. 3U). This result demonstrated that irradiation can activate the Wnt pathway and that LGK-974 can inhibit the Wnt pathway.

LGK-974 treatment increases the radiosensitivity of HepG2 cells by increasing the level of ROS. To determine whether the decrease in Nrf2 protein expression induced by LGK-974 contributes to increased ROS accumulation in HepG2 cells, we measured the intracellular ROS levels after ionizing radiation using the fluorescence indicator 2,7-dichlorodihydrofluorescein diacetate $\left(\mathrm{H}_{2}\right.$ DCFH-DA). We chose $\mathrm{H}_{2} \mathrm{O}_{2}$ treatment as a positive control. The oxidative effect of $\mathrm{H}_{2} \mathrm{O}_{2}$ is well known (30). Irradiation increased the ROS levels in HepG2 cells comparably to $\mathrm{H}_{2} \mathrm{O}_{2}$. We found that the treatment of HepG 2 cells with both $10 \mu \mathrm{M}$ LGK-974 and 4 Gy ionizing radiation clearly increased the levels of ROS after 6 h. Fluorimetric quantification of the change in ROS levels showed that the combination of ionizing radiation and
LGK-974 treatment caused an 2 -2-fold increase in the level of ROS relative to the control treatment and a 1.57 -fold increase relative to ionizing radiation alone. Additionally, LGK-974 alone affected ROS generation (Fig. 4A and B). The increase in ROS levels in the groups treated with ionizing radiation alone or with $\mathrm{H}_{2} \mathrm{O}_{2}$ alone demonstrated that irradiation can increase oxidative stress in cells. To establish whether irradiation or LGK-974 can damage chromosomes, in addition to describing the effect of LGK-974 on DNA, we performed comet assays. HepG2 cells were incubated with or without $10 \mu \mathrm{M}$ LGK-974 for $24 \mathrm{~h}$ and then exposed to $4 \mathrm{~Gy}$ irradiation. The results demonstrated that irradiation damaged the DNA of HepG2 cells and that additional treatment with LGK-974 exacerbated this phenomenon (Fig. 4C,D). In summary, the above results showed that LGK-974 can amplify the effects of radiation on HepG2 cells.

\section{Discussion}

Liver cancer has high malignancy and an insidious onset, is invasive and fast-growing, has high recurrence and mortality rates and is more commonly found in men $(31,32)$. Radiation therapy is an effective treatment method to extend the life of patients with liver cancer $(33,34)$. A major challenge 
$\mathbf{L}$

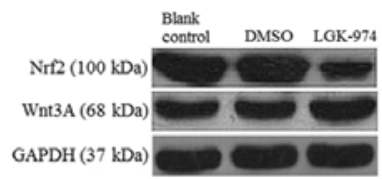

o

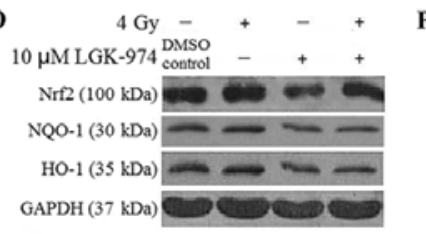

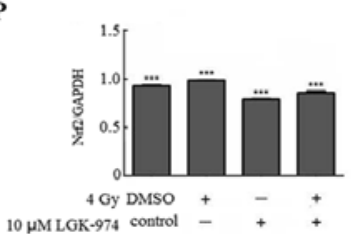
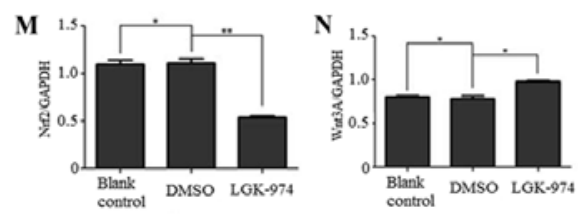

S
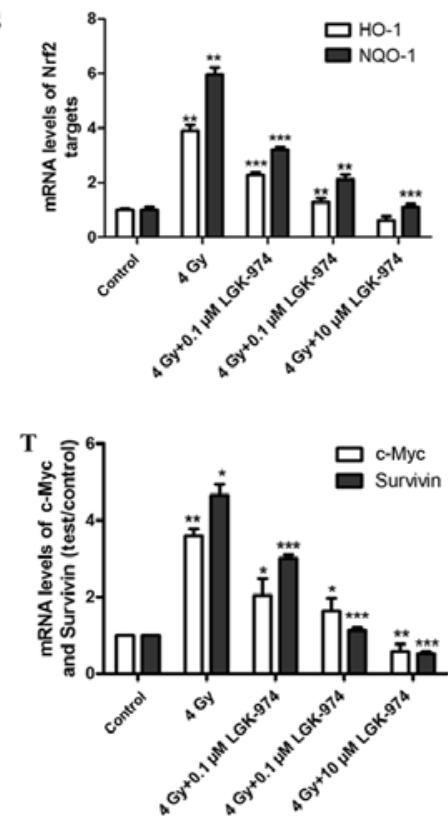

Q

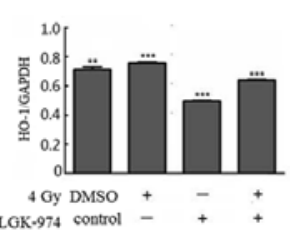

$\mathbf{R}$

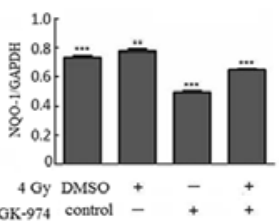

$\mathbf{U}$

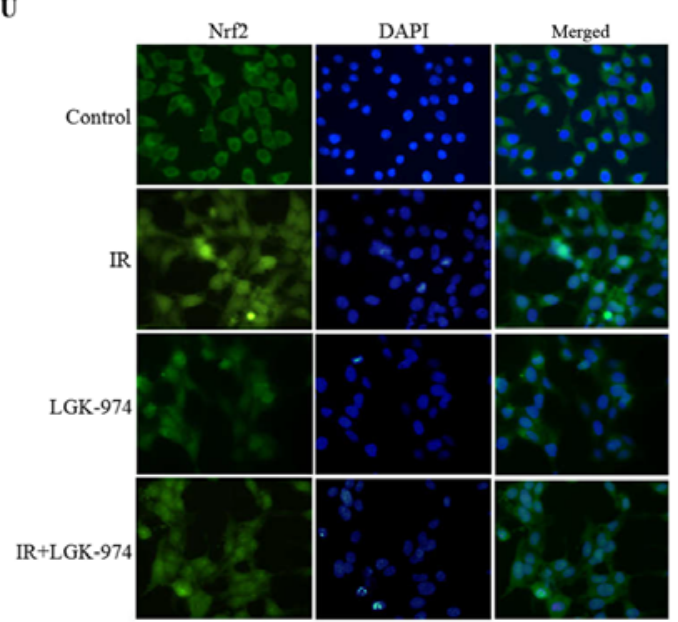

Figure 3. Continued. (L) HepG2 cells were treated with $10 \mu \mathrm{M}$ LGK-974 or solvent DMSO alone. After $24 \mathrm{~h}$, the cells were collected and subjected to western blot analysis. (M and N) Nrf2 and Wnt3A levels in HepG2 cells treated with $10 \mu \mathrm{M}$ LGK-974 or solvent DMSO alone. (O) HepG2 cells were treated with $10 \mu \mathrm{M}$ LGK-974 or exposed to 4 Gy radiation. After 24 h, we collected the cells for western blot analysis. (P-R) Nrf2, HO-1 and NQO-1 levels in HepG2 cells under the different treatments as shown in (O). (S and T) Relative mRNA levels of Nrf2, NQO-1, Wnt3A and $\beta$-catenin in HepG2 cells. HepG2 cells were treated with different concentrations of LGK-974. After $12 \mathrm{~h}$, the cells were exposed to 4 Gy radiation. After an additional $6 \mathrm{~h}$, we collected the cells for total RNA extraction and PCR assays. (U) The Nrf2 levels in different treated HepG2 cells. HepG2 cells were treated with or without $10 \mu \mathrm{M}$ LGK-974 for 24 h; then, the cells were exposed to $4 \mathrm{~Gy}$ irradiation. After an additional $6 \mathrm{~h}$, immunofluorescence analysis was performed.

in the attempt to cure cancer is the radioresistance that is acquired during radiotherapy. Nrf2, a major regulator of redox homeostasis, has previously been confirmed to play an important role in the resistance to oxidative stress during radiation therapy (35). Inhibiting Nrf2 may potentiate cancer radiotherapy (36). Regarding the transcription factor $\mathrm{Nrf} 2$, most studies have focused on the role of the electrophile and redox sensor KEAP1 in modulating NRF2 protein levels to affect cell functions and to exert antioxidative effects. In the present study, we did not examine the Keap1-Nrf2 pathway and instead explored the novel relationship between Wnt and Nrf2. We discovered that LGK-974, a Wnt inhibitor, increases radiosensitivity inhibits Nrf2 signaling. Therefore, Wnt-Nrf2 may represent a novel signaling pathway.

Recently, it has been found that the Wnt signaling pathway and Nrf2 are associated, both in vivo and in vitro (37). The downstream protein complexes of Wnt and Axin1-GSK-3 $\beta$ interact with Nrf2, thus holding Nrf2 in the cytoplasm. Under these conditions, the transcription factor Nrf2 cannot enter the cell nucleus; instead, NRF2 becomes phosphorylated, leading to $\mathrm{b}$-TrCP-dependent ubiquitination and proteasomal degradation. In this situation, antioxidant genes, such as HO-1 and NQO-1, cannot be transcribed. In hepatoma cells, the Wnt classical pathway is initiated when secreted Wnt3A interacts with LRP5/6 and Frizzled embedded in the cell membrane (38). LGK-974 limits the activity of PORCN, which is located in the endoplasmic reticulum and catalyzes Wnt3A protein palmitoylation and secretion (39). $\beta$-catenin is a downstream cytoplasmic protein in the Wnt pathway. In the case of Wnt signaling activation, Wnt escapes degradation, accumulates in the cytoplasm and finally enters the nucleus to induce the transcription of genes such as C-myc. We summarized the 
A
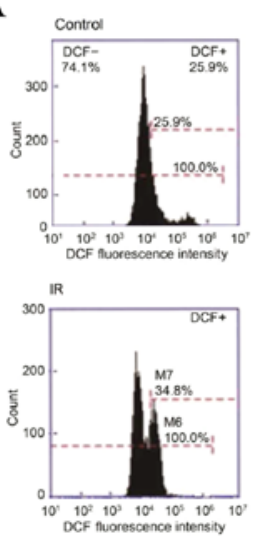

B

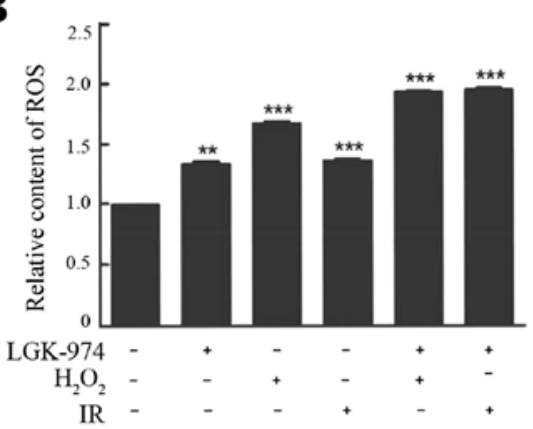

C
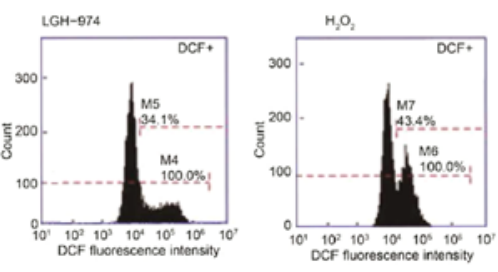

$\mathrm{H}_{2} \mathrm{O},+\mathrm{LGH}-974$
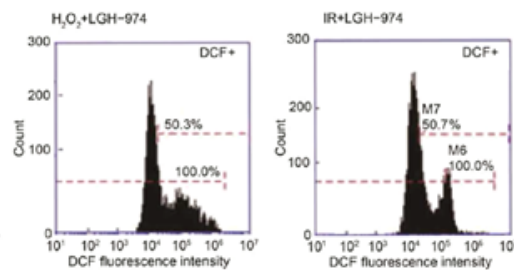

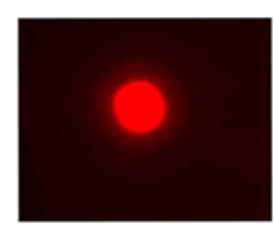

Control

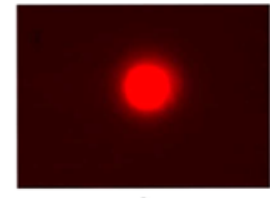

$4 \mathrm{~Gy}$

D

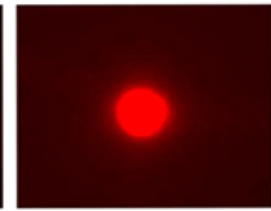

$10 \mu \mathrm{M}+\mathrm{LGK}-974$

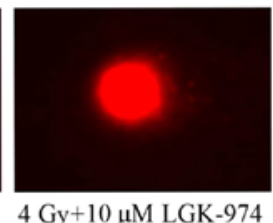

$4 \mathrm{~Gy}+10 \mu \mathrm{M}$ LGK-974

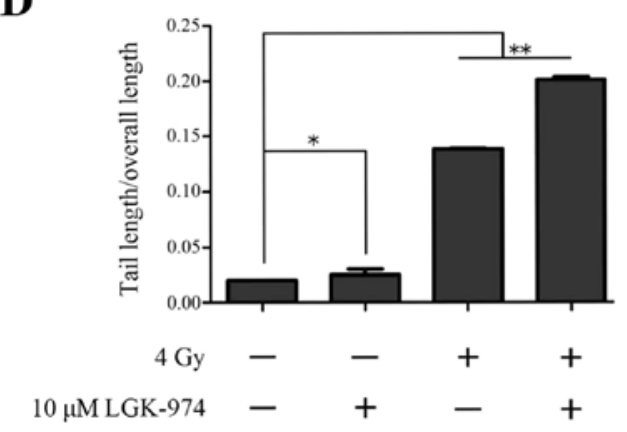

Figure 4. LGK-974 can suppress the resistance of HepG2 cells to oxidative stress. (A and B) HepG2 cells were incubated with $10 \mu \mathrm{M}$ LGK-974 with or without $10 \mu \mathrm{M} \mathrm{H}_{2} \mathrm{O}_{2}$ for $24 \mathrm{~h}$. Cells were incubated with $10 \mu \mathrm{M} \mathrm{LGK}-974$ after $4 \mathrm{~Gy}$ irradiation or exposed to $4 \mathrm{~Gy}$ alone. After $6 \mathrm{~h}$, the ROS levels were evaluated using the fluorescent indicator $\mathrm{H}_{2}$ DCFH-DA. ${ }^{* * *} \mathrm{P}<0.001,{ }^{* *} \mathrm{P}<0.01$. (C and D) HepG2 cells were incubated with or without $10 \mu \mathrm{M}$ LGK-974 for $24 \mathrm{~h}$ and then exposed or not exposed to 4 Gy irradiation.

research findings and utilized the Wnt inhibitor LGK-974 in liver cancer radiotherapy. Preliminarily, we confirmed that LGK-974 has radiosensitizing properties.

As a Wnt inhibitor, LGK-974 has been investigated in several types of cancer. In pancreatic cancer cells, as an inspection control, LGK-974 inhibited cell proliferation and induced cell differentiation in RNF43-mutant pancreatic adenocarcinoma xenograft models (40). In breast cancer cells, blocking the autocrine Wnt signaling pathway using LGK-974 ameliorated NRBP1 overexpression-induced inhibition of cell proliferation (41). LGK974 decreased tumor cell growth and metastasis in 8 head and neck squamous cell carcinoma cell lines (42). LGK-974 also inhibited the proliferation of mucin-producing intrahepatic cholangiocarcinoma and mixed hepatocellular-cholangiocarcinoma (43). All these findings show that LGK-974 can inhibit cancer cell growth and metastasis; however, no previous study has evaluated the effect of LGK-974 on radiosensitivity.

In the present study, a liver cancer cell line was cultured in vitro, and we used the Wnt inhibitor LGK-974 dissolved in DMSO in combination with ionizing radiation to study the possibility that this drug might induce sensitization to radiation. The amount of DMSO applied was very low. As a solvent, DMSO has low toxicity (44), and its effects on cells are far weaker than those of LGK-974. To more clearly demonstrate the effect of LGK-974 on HepG2 cells, we compared the experimental groups with a group of HepG2 cells cultured under normal conditions. Our results showed that
LGK-974 significantly inhibited the growth of HepG2 cells and promoted cell apoptosis. LGK-974 inhibited C-myc gene expression downstream of Wnt3A, NQO-1 gene expression downstream of Nrf2, and HO-1 gene and protein expression. Analysis of the ROS levels in HepG2 cells revealed that the antioxidant capacity of these cells decreased in the presence of LGK-974.

At present, our studies were performed only at the cellular level. In addition, only one cell line was used for mechanistic studies. Further experiments should be performed on more cell lines, animal models and subsequently, humans. To further elucidate the mechanism of action of LGK-974, we will perform additional assays, as the findings from the present study were not comprehensive. Although we performed flow cytometry to examine cell apoptosis, this single assay is inadequate. We will perform TUNEL staining to obtain images of apoptotic cells in future research. LGK-974 is an oral medicine that has already been accepted for phase I clinical trials, although only as a tumor chemotherapy drug, not for applications involving radiotherapy. This inhibitor of Wnt can also block Nrf2 function in HepG2 cells, suggesting that Wnt-Nrf2 may be a novel pathway related to radiation oncology. The discovery of the radiosensitizing effect of LGK-974 has great value. Once applied in the clinic, this treatment may be beneficial to liver cancer patients.

LGK-974 is an effective inhibitor of the canonical Wnt signaling pathway. We demonstrated that LGK-974 can inhibit Nrf2 signaling by limiting the entry of Nrf2 into the nucleus. 
LGK-974 has cytotoxicity and can increase the radiosensitivity of HepG2 cells. These findings provide new ideas for novel potential radiosensitizing drugs in cancer radiotherapy.

\section{Acknowledgements}

The present study was supported by the National Natural Science Foundation of China (nos. 31670859 and 81470098); the Natural Science Foundation of Tianjin (no. 15KPXM01SF056); the Fundamental Research Funds from CAMS and PUMC (no. 2016ZX310068); and the Research Funds for the Innovation Team of IRM-CAMS (no. 1650).

\section{References}

1. Torre LA, Bray F, Siegel RL, Ferlay J, Lortet-Tieulent J and Jemal A: Global cancer statistics, 2012. CA Cancer J Clin 65: 87-108, 2015

2. Teltscharov L, Vlachov K and Marovski T: Effect of radiotherapy on the liver function of cancer patients. Radiobiol Radiother (Berl) 5: 673-677, 1964 (In German).

3. He L, Lai H and Chen T: Dual-function nanosystem for synergetic cancer chemo-/radiotherapy through ROS-mediated signaling pathways. Biomaterials 51: 30-42, 2015.

4. DeNicola GM, Karreth FA, Humpton TJ, Gopinathan A, Wei C, Frese K, Mangal D, Yu KH, Yeo CJ, Calhoun ES, et al: Oncogeneinduced Nrf2 transcription promotes ROS detoxification and tumorigenesis. Nature 475: 106-109, 2011.

5. Merchant AA, Singh A, Matsui W and Biswal S: The redox-sensitive transcription factor Nrf2 regulates murine hematopoietic stem cell survival independently of ROS levels. Blood 118: 6572-6579, 2011

6. Hayes JD and Dinkova-Kostova AT: The Nrf2 regulatory network provides an interface between redox and intermediary metabolism. Trends Biochem Sci 39: 199-218, 2014.

7. Kitteringham NR, Abdullah A, Walsh J, Randle L, Jenkins RE, Sison R, Goldring CE, Powell H, Sanderson C, Williams S, et al: Proteomic analysis of Nrf2 deficient transgenic mice reveals cellular defence and lipid metabolism as primary Nrf2-dependent pathways in the liver. J Proteomics 73: 1612-1631, 2010.

8. Mitsuishi Y, Taguchi K, Kawatani Y, Shibata T, Nukiwa T, Aburatani H, Yamamoto $M$ and Motohashi H: Nrf2 redirects glucose and glutamine into anabolic pathways in metabolic reprogramming. Cancer Cell 22: 66-79, 2012.

9. Wu KC, Cui JY and Klaassen CD: Effect of graded Nrf2 activation on phase-I and -II drug metabolizing enzymes and transporters in mouse liver. PLoS One 7: e39006, 2012.

10. Saw CL, Wu Q and Kong AN: Anti-cancer and potential chemopreventive actions of ginseng by activating Nrf2 (NFE2L2) anti-oxidative stress/anti-inflammatory pathways. Chin Med 5: 37, 2010.

11. Seng S, Avraham HK, Jiang S, Yang S, Sekine M, Kimelman N, $\mathrm{Li} \mathrm{H}$ and Avraham S: The nuclear matrix protein, NRP/B, enhances Nrf2-mediated oxidative stress responses in breast cancer cells. Cancer Res 67: 8596-8604, 2007.

12. Hong CC, Ambrosone CB, Ahn J, Choi JY, McCullough ML, Stevens VL, Rodriguez C, Thun MJ and Calle EE: Genetic variability in iron-related oxidative stress pathways (Nrf2, NQ01, NOS3, and HO-1), iron intake, and risk of postmenopausal breast cancer. Cancer Epidemiol Biomarkers Prev 16: 1784-1794, 2007.

13. Zipper LM and Mulcahy RT: The Keap1 BTB/POZ dimerization function is required to sequester Nrf2 in cytoplasm. J Biol Chem 277: 36544-36552, 2002.

14. Nasiri HR, Linge S and Ullmann D: Thermodynamic profiling of inhibitors of Nrf2:Keap1 interactions. Bioorg Med Chem Lett 26: 526-529, 2016.

15. Leinonen HM, Kansanen E, Pölönen P, Heinäniemi M and Levonen AL: Dysregulation of the Keap1-Nrf2 pathway in cancer. Biochem Soc Trans 43: 645-649, 2015.

16. McMahon M, Thomas N, Itoh K, Yamamoto M and Hayes JD: Dimerization of substrate adaptors can facilitate cullin-mediated ubiquitylation of proteins by a 'tethering' mechanism: A two-site interaction model for the Nrf2-Keap1 complex. J Biol Chem 281: 24756-24768, 2006.
17. Tong KI, Kobayashi A, Katsuoka F and Yamamoto M: Two-site substrate recognition model for the Keap1-Nrf2 system: A hinge and latch mechanism. Biol Chem 387: 1311-1320, 2006.

18. Wang C, Li C, Peng H, Ye Z, Zhang J, Liu X and Lou T: Activation of the Nrf2-ARE pathway attenuates hyperglycemia-mediated injuries in mouse podocytes. Cell Physiol Biochem 34: 891-902, 2014.

19. Lee LY, Köhler UA, Zhang L, Roenneburg D, Werner S, Johnson JA and Foley DP: Activation of the Nrf2-ARE pathway in hepatocytes protects against steatosis in nutritionally induced non-alcoholic steatohepatitis in mice. Toxicol Sci 142: 361-374, 2014.

20. Rada P, Rojo AI, Offergeld A, Feng GJ, Velasco-Martín JP, González-Sancho JM, Valverde ÁM, Dale T, Regadera J and Cuadrado A: WNT-3A regulates an Axin1/NRF2 complex that regulates antioxidant metabolism in hepatocytes. Antioxid Redox Signal 22: 555-571, 2015.

21. MacDonald BT, Semenov MV, Huang H and He X: Dissecting molecular differences between Wnt coreceptors LRP5 and LRP6. PLoS One 6: e23537, 2011.

22. Mi K and Johnson GV: Role of the intracellular domains of LRP5 and LRP6 in activating the Wnt canonical pathway. J Cell Biochem 95: 328-338, 2005.

23. Zeng X, Tamai K, Doble B, Li S, Huang H, Habas R, Okamura H, Woodgett $\mathrm{J}$ and $\mathrm{He} \mathrm{X}$ : A dual-kinase mechanism for Wnt co-receptor phosphorylation and activation. Nature 438: 873-877, 2005.

24. MacDonald BT, Yokota C, Tamai K, Zeng X and He X: Wnt signal amplification via activity, cooperativity, and regulation of multiple intracellular PPPSP motifs in the Wnt co-receptor LRP6. J Biol Chem 283: 16115-16123, 2008.

25. Kim M, Kim S, Lee SH, Kim W, Sohn MJ, Kim HS, Kim J and Jho EH: Merlin inhibits Wnt/ $\beta$-catenin signaling by blocking LRP6 phosphorylation. Cell Death Differ 23: 1638-1647, 2016.

26. Morgan JT, Raghunathan VK, Chang YR, Murphy CJ and Russell P: Wnt inhibition induces persistent increases in intrinsic stiffness of human trabecular meshwork cells. Exp Eye Res 132: 174-178, 2015.

27. Abrami L, Kunz B, Iacovache I and van der Goot FG: Palmitoylation and ubiquitination regulate exit of the Wnt signaling protein LRP6 from the endoplasmic reticulum. Proc Natl Acad Sci USA 105: 5384-5389, 2008.

28. Komekado H, Yamamoto $H$, Chiba $T$ and Kikuchi A: Glycosylation and palmitoylation of Wnt-3a are coupled to produce an active form of Wnt-3a. Genes Cells 12: 521-534, 2007.

29. Kurayoshi M, Yamamoto H, Izumi S and Kikuchi A: Posttranslational palmitoylation and glycosylation of Wnt-5a are necessary for its signalling. Biochem J 402: 515-523, 2007.

30. Rhee SG: Redox signaling: Hydrogen peroxide as intracellular messenger. Exp Mol Med 31: 53-59, 1999.

31. Chen W, Zheng R, Baade PD, Zhang S, Zeng H, Bray F, Jemal A, $\mathrm{Yu}$ XQ and He J: Cancer statistics in China, 2015. CA Cancer J Clin 66: 115-132, 2016.

32. Siegel R, Naishadham D and Jemal A: Cancer statistics for Hispanics/Latinos, 2012. CA Cancer J Clin 62: 283-298, 2012.

33. Hosoda Y, Kim Y, Nishino M, Okano M, Nagai K, Yasui M and Tsujinaka T: A case of rectal cancer successfully treated with stereotactic radiotherapy for liver and lung metastases. Gan To Kagaku Ryoho 42: 2109-2111, 2015 (In Japanese).

34. Tono T, Hashimoto K, Yamada Y, Nishida K, Yanagawa T, Danno K, Fujie Y, Fujita S, Fujita J, Yoshida T, et al: Efficacy of stereotactic radiotherapy for primary and metastatic liver cancer. Gan To Kagaku Ryoho 40: 1853-1855, 2013 (In Japanese).

35. Zhou S, Ye W, Shao Q, Zhang M and Liang J: Nrf2 is a potential therapeutic target in radioresistance in human cancer. Crit Rev Oncol Hematol 88: 706-715, 2013.

36. Menegon S, Columbano A and Giordano S: The dual roles of NRF2 in cancer. Trends Mol Med 22: 578-593, 2016.

37. Nault JC, Rebouissou S and Zucman Rossi J: NRF2/KEAP1 and $W n t / \beta$-catenin in the multistep process of liver carcinogenesis in humans and rats. Hepatology 62: 677-679, 2015.

38. Go GW, Srivastava R, Hernandez-Ono A, Gang G, Smith SB Booth CJ, Ginsberg HN and Mani A: The combined hyperlipidemia caused by impaired Wnt-LRP6 signaling is reversed by Wnt3a rescue. Cell Metab 19: 209-220, 2014. 
39. Madan B, Ke Z, Harmston N, Ho SY, Frois AO, Alam J, Jeyaraj DA, Pendharkar V, Ghosh K, Virshup IH, et al: Wnt addiction of genetically defined cancers reversed by PORCN inhibition. Oncogene 35: 2197-2207, 2016.

40. Jiang X, Hao HX, Growney JD, Woolfenden S, Bottiglio C, Ng N, Lu B, Hsieh MH, Bagdasarian L, Meyer R, et al: Inactivating mutations of RNF43 confer Wnt dependency in pancreatic ductal adenocarcinoma. Proc Natl Acad Sci USA 110: 12649-12654, 2013.

41. Wei H, Wang H, Ji Q, Sun J, Tao L and Zhou X: NRBP1 is downregulated in breast cancer and NRBP1 overexpression inhibits cancer cell proliferation through $\mathrm{Wnt} / \beta$-catenin signaling pathway. Onco Targets Ther 8: 3721-3730, 2015.
42. Rudy SF, Brenner JC, Harris JL, Liu J, Che J, Scott MV, Owen JH, Komarck CM, Graham MP, Bellile EL, et al: In vivo Wnt pathway inhibition of human squamous cell carcinoma growth and metastasis in the chick chorioallantoic model. J Otolaryngol Head Neck Surg 45: 26, 2016.

43. Fraveto A, Cardinale V, Bragazzi MC, Giuliante F, De Rose AM, Grazi GL, Napoletano C, Semeraro R, Lustri AM, Costantini D, et al: Sensitivity of human intrahepatic cholangiocarcinoma subtypes to chemotherapeutics and molecular targeted agents: A study on primary cell cultures. PLoS One 10: $\mathrm{e} 0142124,2015$.

44. Galvao J, Davis B, Tilley M, Normando E, Duchen MR and Cordeiro MF: Unexpected low-dose toxicity of the universal solvent DMSO. FASEB J 28: 1317-1330, 2014. 